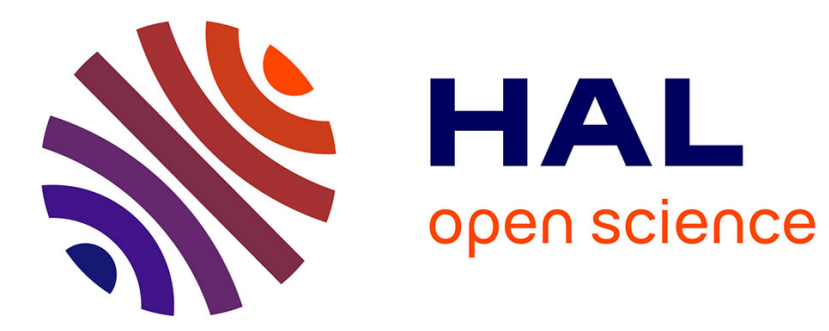

\title{
Co-Limbs: An Intuitive Collaborative Control for Wearable Robotic Arms
}

Guillaume Gourmelen, Adrien Verhulst, Tomoya Sasaki, Benjamin Navarro, Gowrishankar Ganesh, Masahiko Inami

\section{- To cite this version:}

Guillaume Gourmelen, Adrien Verhulst, Tomoya Sasaki, Benjamin Navarro, Gowrishankar Ganesh, et al.. Co-Limbs: An Intuitive Collaborative Control for Wearable Robotic Arms. SA 2019 - 12th ACM SIGGRAPH Conference and Exhibition on Computer Graphics and Interactive Techniques in Asia, Nov 2019, Brisbane, Australia. pp.9-10, 10.1145/3355049.3360526 . hal-02407032

\section{HAL Id: hal-02407032 \\ https://hal.science/hal-02407032}

Submitted on 18 Sep 2020

HAL is a multi-disciplinary open access archive for the deposit and dissemination of scientific research documents, whether they are published or not. The documents may come from teaching and research institutions in France or abroad, or from public or private research centers.
L'archive ouverte pluridisciplinaire HAL, est destinée au dépôt et à la diffusion de documents scientifiques de niveau recherche, publiés ou non, émanant des établissements d'enseignement et de recherche français ou étrangers, des laboratoires publics ou privés. 


\section{Co-Limbs: An Intuitive Collaborative Control for Wearable Robotic Arms}

\author{
Guillaume Gourmelen* \\ CNRS-UM LIRMM(1), France \\ guillaume.gourmelen@lirmm.fr \\ Benjamin Navarro \\ CNRS-UM LIRMM(1), France \\ benjamin.navarro@lirmm.fr
}

\author{
Adrien Verhulst* \\ RCAST Univ. Tokyo(2), Japan \\ adrienverhulst@star.rcast.u-tokyo.ac.jp \\ Gowrishankar Ganesh \\ CNRS-UM LIRMM(1), France \\ ganesh.gowrishankar@lirmm.fr
}

\author{
Tomoya Sasaki* \\ RCAST Univ. Tokyo(2), Japan \\ sasaki@star.rcast.u-tokyo.ac.jp \\ Masahiko Inami \\ RCAST Univ. Tokyo(2), Japan \\ inami@star.rcast.u-tokyo.ac.jp
}

\begin{abstract}
The promising possibilities offered by supernumerary robotic wearable arms are limited by the lack of an intuitive and robust user interface to control them. Here, utilizing admittance control, we propose a 'Collaborative limbs', or 'Co-limbs' user interface for wearable robot arms. The key feature of this user interface is its intuitiveness enabling even first time users to immediately move and use the, normally stiff, robot arms for assistive tasks and even teach the robot simple and useful movements. We demonstrate the diverse range of applications enabled by this simple but powerful user interface through example demonstrations in the Passive Assist, Power Assist and Playback modes.
\end{abstract}

\section{CCS CONCEPTS}

- Computer systems organization $\rightarrow$ Embedded systems; Redundancy; Robotics.

\section{KEYWORDS}

Robotic Supernumerary limbs, Collaborative control, assitive robotics, Human-robot interaction

\section{INTRODUCTION}

Imagine if you had two extra robotic arms to enable you to pull your luggage in the airport and hold your coffee cup while you take the hand of your child, or to assist you in holding a heavy box, or even to wave a hand fan back and forth on a hot day while you read a newspaper. These promising and exciting possibilities have led to the development of supernumerary Wearable Robotic Arms (WRA) by several groups [??], including ours [?]. Yet, these designs remain limited to demonstrations, and have not found commercial popularity because of the lack of intuitive and efficient user interface to operate them.

A good user interface should be intuitive to use and should enable the user to easily convey to the robot what he/she wants, and how to achieve it. The first requirement for any operation with the robot arm (like holding a cup, pulling the luggage, etc) is for it to be positioned and oriented appropriately. However, even this first operation is difficult in the current WRA because of the popular use of servo motors in these wearable systems- which helps reduce their

*(1)=Laboratoire d'Informatique, de Robotique et de Microelectronique de Montpellier; (2)=Research Center for Advanced Science and Technology, The University of Tokyo

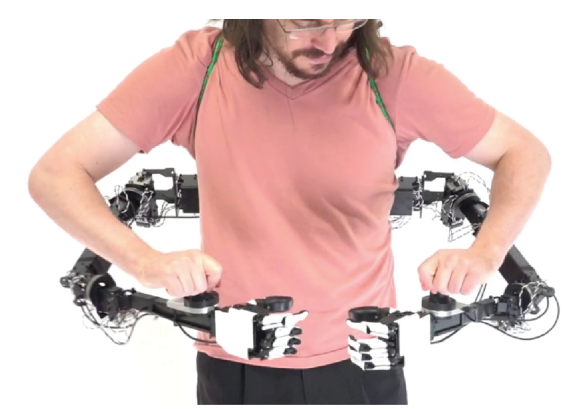

Figure 1: The Co-Limbs user interface uses force sensors and admittance control to enable a user to intuitively move and use the otherwise non-backdrivable wearable robotic arms.

weight, but the high gear ratios in these motors results in the robot being stiff and servo control makes the robot non-back-drivable. Positioning the robot arms thus requires the use of either joysticks, or tracking of other limbs, which, due to positioning being in 12 dimensional space ( 3 position +3 orientations $) \times 2$ arms) and over a large workspace around the user's body, can require significant visual attention and time. To address this issue, here we propose a simple but promising user interface, called 'Co-limbs', that is based on the idea of collaborative control [?] in which the user and robot work on the task together. This interface is intuitive to use enabling even a first time user to immediately position, orient and use the device in multiple scenarios, and even teach the WRA simple movements.

\section{CO-LIMB INTERFACE DESCRIPTION}

Humans are adept in embodying and using hand-held tools [??]. They can immediately position and orient a hand held tool as per task requirements, often requiring minimal visual feedback in order to do so. We therefore hypothesized that an interface that promotes the WRA to be viewed as tools by the user, would automatically make it easy for the user to operate them. This however, is not directly possible in non-backdrivable system. The Co-limb system thus proposes to have a handle equipped with a force sensor ${ }^{1}$ on the forearm of each WRA and utilize admittance control to allow the user to move the non-backdrivable arms. In admittance control, the torque wrench $W$ applied on each handle is recorded

\footnotetext{
${ }^{1}$ We use two Leptrino 6 Axis Force Sensor (055YA 501)
} 
in the force sensor coordinates $\left(W_{f \text { sensor }}\right)$, and, knowing the current pose of the robot arm, transformed in the world space coordinates $\left(T^{\text {world }}(Q)_{\text {fsensor }}\right)$, with $Q$ a 7-dimensional vector of the current joint states of each arm The force is then converted to a corresponding velocity in world space with an assumed dynamics $\omega_{\text {world }}=\delta\left(m, d, W_{\text {world }}\right)$, with mass $m$ and damping $d$. Finally the joint velocities is calculated using inverse kinematics as $\omega_{\text {world }}=I K\left(\omega_{\text {world }}, Q\right)$. Our current application is implemented in Linux using $m=1 \mathrm{~kg}, d=15 \mathrm{~kg} / \mathrm{m}$ and the inverse kinematics functionality provided by the openHRI package developed in our $\mathrm{lab}^{2}$.

\subsection{Main features}

Our relatively simple interface has several salient features that radically expand the applications of a WRA systems.

- The key feature of our interface is its intuitiveness, enabling even a first time user to use it immediately with no training.

- Our interface enables back-drivability, a feature key for user comfort, and enabling the user to easily position the device for applications of passive assistive.

- Collaborative guidance: Two fundamental challenges for a robot interacting with a human is to (1) understand the intention of the user, and (2) to plan its own movements to help the user accordingly. Our interface proposes to overcome these issues by allowing the user to collaboratively guide the robot, while benefiting from the power assistance [?].

- Better teaching:The collaborative guidance opens up the possibility of using teaching by demonstration [?] techniques to improve the skills of the WRA (c.f. demonstration video).

- Versatility: While we do not show this in our current demonstration, the user interface also allows us to modulate the dynamics felt by the user, allowing us to make the WRA feel heavy or light, use the robot system to cancel possible tremors and noise in the user input (for example when the system is used by a elderly user)

\section{POSSIBLE APPLICATION}

We demonstrate the intuitiveness and versatility enabled by our proposed user interface in 3 example modes of application utilizing our WRA device [?]. $\bigcirc$ First, the Passive Assist mode allows the user to orient the robot hands in the desired postures and to utilize them in given scenario (e.g. pull a suitcase or hold an umbrella, c.f. video). The 1 DoF robot hand (open or close fingers) is activated by a switch on the handle. Second, the Power Assist mode allows the user to guide the robot arms and pick up cumbersome loads (e.g. lifting a big box, c.f. video). Note that the weight of the load is obviously transferred to the user (as the robot system is worn by the user), but the robot distributes the load over the back and waist making it more comfortable than lifting the load with their hands. The Power Assist mode can be particularly useful for elderly users as it can assist their own body weight, for example, assist them in standing up. $\bigcirc$ Third, the Playback mode allows the users to record and playback simple but useful repetitive movements to

OpenPHRI, a complete and generic solution for safe physical human-robot interactions, Benjamin Navarro, 2018 the robot, so that the robot can then perform them without user guidance (e.g. use a hand held fan, c.f. video).

\section{CONCLUSION}

In this study, we introduce a simple user interface for supernumerary wearable robot arm systems. This interface utilizes a force sensor to enable users to guide the robot arms and enable various passive and active assistance tasks, and enabling the user to teach simple movements to the robot. The key feature of this user interface is its intuitiveness and ease of use. We currently demonstrate the versatility of the interface in three modes of application, and we are now developing its applications in the field of elderly care where we believe it can be extremely useful.

\section{ACKNOWLEDGMENTS}

This project was supported by JST ERATO Grant Number JPMJER1701, Japan.

We thank MHD Yamen Saraiji who provided sample source code for our prototype. 